\title{
THE NUMBER OF WEAKLY COMPACT SETS WHICH GENERATE A BANACH SPACE
}

\author{
ANTONIO AVILÉS
}

\begin{abstract}
We consider the cardinal invariant $C G(X)$ of the minimal number of weakly compact subsets which generate a Banach space $X$. We study the behavior of this index when passing to subspaces, its relation with the Lindelöf number in the weak topology and other related questions.
\end{abstract}

A Banach space is weakly compactly generated if there is a weakly compact subset which is linearly dense and weakly Lindelöf if it is a Lindelöf space in its weak topology. It was asked by Corson [10] which was the relation between these two concepts. The answer was that every weakly compactly generated space is weakly Lindelöf but the converse is not true, and in order to clarify what was in the middle the class of weakly $\mathcal{K}$-analytic was introduced by Talagrand [18, who was together with Pol [15] the first to solve this problem. Here we shall analyze the question of Corson from a more general point of view: What is the relation between the number of weak compacta which are necessary to generate a Banach space and the Lindelöf number of the space in the weak topology? Again, an intermediate class analagous to that introduced by Talagrand plays a clarifying role in the theory. Thus, our starting point is the following (cf. Sections 1 and 2 for notation):

Definition 1. Let $X$ be a topological space.

(1) The index of compact generation of $X, C G(X)$, is defined as the least infinite cardinal $\kappa$ such that there exists a family $\left\{K_{\lambda}: \lambda<\kappa\right\}$ of compact subsets of $X$ whose union is a dense subset of $X$.

(2) The index of $\mathcal{K}$-analyticity of $X, \ell K(X)$, is the least infinite cardinal $\kappa$ for which there exists a complete metric space $M$ of weight $\kappa$ and an usco $M \longrightarrow 2^{X}$.

(3) The Lindelöf number of $X, \ell(X)$, is the least infinite cardinal $\kappa$ such that any cover of $X$ by open sets has a subcover with at most $\kappa$ many sets.

If $X$ is a Banach space, all the indices will refer always to the weak topology of $X$. In this way the classes of weakly compactly generated, weakly $\mathcal{K}$-analytic and weakly Lindelöf Banach spaces equal the classes of spaces $X$ such that $C G(X)=$ $\omega, \ell K(X)=\omega$ and $\ell(X)=\omega$ respectively. Similar indices to $\ell K(X)$ can be defined if instead of complete metric spaces of a given weight we use other classes of topological spaces. These kind of indices have been studied in [13, cf. [9], such as the index of $\mathcal{K}$-determinacy $\ell \Sigma(X)$ (taking in (2) arbitrary metric spaces of weight $\kappa$ instead of complete metric spaces) and the Nagami index $N a g(X)$ (taking

2000 Mathematics Subject Classification. 46B26.

This research was partially supported by the grant BFM2002-01719 of MCyT (Spain) and a FPU grant of MEC (Spain). 
arbitrary completely regular topological spaces of weight $\kappa$ ). For any Banach space $X$ we have that

$$
\ell(X) \leq N a g(X) \leq \ell \Sigma(X) \leq \ell K(X) \leq C G(X)
$$

The first inequality can be found in $[9]$ and follows from the fact that the Lindelöf number cannot increase by an usco image. The others are self-evident excepts perhaps the last one, cf. Section 6 below. This gives a first answer to our original question: the number of weak compacta which are necessary to generate a Banach space is not lower than the Lindelöf number in the weak topology, shortly $\ell(X) \leq$ $C G(X)$. On the other hand, we shall show that $C G(X)$ may be arbitrarily larger than $\ell(X)$, namely in Section 5 we prove:

Theorem 2. Let $\kappa$ be any cardinal. There exists a weakly Lindelöf determined Banach space $X$ such that $C G(X)>\kappa$.

Weakly Lindelöf determined spaces constitute a special class of weakly Lindelöf Banach spaces [2. The relation of $C G(X)$ with the other indices is quite different because the cardinality of a completely regular space of weight $\kappa$ is at most $2^{\kappa}$, so $C G(X) \leq 2^{\operatorname{Nag}(X)}$, and by the same reason $C G(X) \leq \ell \Sigma(X)^{\omega}$.

Another classical question is the fact, first shown by Rosenthal [17, that there are subspaces of weakly compactly generated spaces which are not weakly compactly generated. It has been observed in [5] that such spaces must have large enough density character, namely greater than or equal to cardinal b. In Section 7, we address the natural question now: What is the relation between $C G(X)$ and $C G(Y)$ for $Y$ a subspace of $X$ ? and what about the density character? The answer we give is the following:

Theorem 3. Let $\kappa, \tau, \delta$ be infinite cardinals. The following are equivalent:

(1) $\tau \leq \mathbf{d}(\kappa)$ and $\delta \geq \mathbf{b}_{\kappa}(\tau)$.

(2) There exists a Banach space $X$ and a subspace $Y$ of $X$ such that $C G(X)=$ $\kappa, C G(Y)=\tau$ and $\operatorname{dens}(Y)=\delta$.

The cardinal numbers $\mathbf{d}(\kappa)$ and $\mathbf{b}_{\kappa}(\tau)$ are defined in Section 1 in terms of the topology of the space $\kappa^{\omega}$. For cardinals $\kappa, \tau \geq 2^{\omega}$, it happens that $\mathbf{d}(\kappa)=\kappa^{\omega}$ and $\mathbf{b}_{\kappa}(\tau)=\tau$ but for cardinals below the continuum the behavior of this functions is more complicated and depends heavily on the axiomatic settlement. The fact that (1) implies (2) in Theorem 3 is obtained by modifying an example of Argyros [11, Section 1.6] while the converse is based on the use of the index $\mathcal{K}$-analyticity. It is indeed established in Section $[6$ a very similar result to Theorem 3 concerning the relation of the indices $\ell K(X)$ and $C G(X)$ :

Theorem 4. Let $\kappa, \tau, \delta$ be infinite cardinals. The following are equivalent:

(1) $\kappa \leq \tau \leq \mathbf{d}(\kappa)$ and $\delta \geq \mathbf{b}_{\kappa}(\tau)$.

(2) There exists a Banach space $X$ such that $\ell K(X)=\kappa, C G(X)=\tau$ and $\operatorname{dens}(X)=\delta$.

Here, the fact that (1) implies (2) is obtained by modifying the construction of Talagrand [18] of a weakly $\mathcal{K}$-analytic space which is not weakly compactly generated. It remains unclear to us what are the precise relations between the indices $\ell K(X), \ell \Sigma(X)$ and $N a g(X)$ for Banach spaces. We make some remarks about that 
in Section 8

We want to express our gratitude to Bernardo Cascales and José Orihuela for their help and support during this work, as well as to David Fremlin for valuable comments and suggestions.

\section{Cardinal numbers and metric spaces}

In this section we fix the notation about cardinal arithmetic and metric spaces, and we shall define and discuss some cardinal numbers which will be used in Sections 6 and 7. A cardinal number $\kappa$ is identified with the set of all ordinals less than $\kappa$, and in particular $\kappa$ is a set of cardinality $\kappa$, and is considered also as a topological space endowed with the discrete topology. By $\kappa^{\omega}$ we denote the set of all sequences of elements of $\kappa$ endowed with the product topology (with respect to the discrete topology on each factor), as well as the cardinality of this set. Finally, $2^{A}$ stands for the family of all subsets of $A$, and when $A=\kappa$ is a cardinal, $2^{\kappa}$ also denotes the cardinality of this set.

Definition 5. Let $\kappa$ be a cardinal number:

(1) The cardinal $\mathbf{d}(\kappa)$ is defined as the least cardinal $\lambda$ such that $\kappa^{\omega}$ is the union of $\lambda$ many compact subsets.

(2) Let $\tau$ be a cardinal such that $\tau \leq \mathbf{d}(\kappa)$. The cardinal $\mathbf{b}_{\kappa}(\tau)$ is the least cardinal $\lambda$ for which there exists a set $A$ of cardinality $\lambda$ such that $A$ is not contained in any union of less than $\tau$ many compact subsets of $\kappa^{\omega}$.

Notice that always $\kappa \leq \mathbf{d}(\kappa) \leq \kappa^{\omega}$ and $\tau \leq \mathbf{b}_{\kappa}(\tau) \leq \kappa^{\omega}$, and that $\mathbf{b}_{\kappa}(\tau)=\tau$ if $\tau \leq \kappa$ (we can consider $A$ a closed and discrete subset of $\kappa^{\omega}$ of cardinality $\tau$ ). Every compact metrizable space is either countable or has cardinality $2^{\omega}$, hence $\mathbf{d}(\kappa)=\kappa^{\omega}$ whenever $\kappa>2^{\omega}$ and $\mathbf{b}_{\kappa}(\tau)=\tau$ whenever $2^{\omega}<\tau \leq \mathbf{d}(\kappa)$. On the other hand, if $\operatorname{cof}(\kappa)>\omega$ then $\kappa^{\omega}=\bigcup_{\alpha<\kappa} \alpha^{\omega}$, and this fact implies that $\mathbf{d}(\kappa)=\sum_{\alpha<\kappa} \mathbf{d}(|\alpha|)$. The difficult case in computing $\mathbf{d}(\kappa)$ is when $\kappa$ is a cardinal of cofinality $\omega$ less than $2^{\omega}$. For example, when $\kappa=\omega$ we refer to 20] for information about cardinal $\mathbf{d}=\mathbf{d}(\omega)$. We illustrate also the situation for $\kappa=\omega_{\omega}$, for which we need the following observation, pointed out to us by David Fremlin:

Proposition 6. For an infinite cardinal $\kappa, \mathbf{d}(\kappa)=\max [\mathbf{d}, \operatorname{cf}([\kappa] \leq \omega)]$, where $\operatorname{cf}([\kappa] \leq \omega)$ is the least cardinality of a cofinal family $A$ of countable subsets of $\kappa$, that is, a family such that every countable subset of $\kappa$ is a subset of some member of $A$.

We mention that Shelah has obtained that $\operatorname{cf}\left(\left[\omega_{\omega}\right] \leq \omega\right)<\omega_{\omega_{4}}$, cf. $[8$. The proof of Proposition 6 is not difficult: if $B$ is a family of compact sets covering $\kappa^{\omega}$, then the family $A=\left\{\left\{x_{n}: x=\left(x_{i}\right)_{i<\omega} \in K, n<\omega\right\}: K \in B\right\}$ is a cofinal family of countable subsets of $\kappa$, and conversely if $A$ is a cofinal family of countable subsets of $\kappa$ and for every $s \in A, C_{s}$ is a family of $\mathbf{d}$ many compact sets covering $s^{\omega}$, then $B=\bigcup_{s \in A} C_{s}$ is a family of compact sets covering $\kappa^{\omega}$.

About cardinals $\mathbf{b}_{\kappa}(\tau)$ we know very little more except for $\mathbf{b}=\mathbf{b}_{\omega}\left(\omega_{1}\right)$ [20] and the fact that in some cases we can establish a relation with the known $\mathbf{b}$, for example $\mathbf{b} \leq \mathbf{b}_{\omega}\left(\omega_{2}\right)=\mathbf{b}_{\omega_{1}}\left(\omega_{2}\right)$ provided $\omega_{1}<\mathbf{d}$. 


\section{The $\mathcal{K}$-AnALYTICITY INDEX}

If $\Sigma$ and $Y$ are topological spaces, we will say that a map $\phi: \Sigma \longrightarrow 2^{Y}$ is an usco if the three following conditions are satisfied:

(1) $\phi(\sigma)$ is a compact subset of $Y$, for every $\sigma \in \Sigma$.

(2) For every open subset $U$ of $Y$, the set $\{\sigma \in \Sigma: \phi(\sigma) \subseteq U\}$ is an open subset of $\Sigma$.

(3) $\bigcup_{\sigma \in \Sigma} \phi(\sigma)=Y$.

In this situation, if $A$ is a subset of $\Sigma$, we denote $\phi(A)=\bigcup\{\phi(\sigma): \sigma \in A\}$.

We recall that any complete metric space $X$ of weight $\kappa$ is a continuous image of a closed subset $M$ of $\kappa^{\omega}$ : One considers a base $\left\{O_{\lambda}: \lambda<\kappa\right\}$ of $X$ and $M=\{x \in$ $\left.\kappa^{\omega}: \operatorname{diam}\left(O_{x_{n}}\right)<\frac{1}{n}, \overline{O_{x_{n+1}}} \subset O_{x_{n}}\right\}$. In addition, for $M$ a closed subset of $\kappa^{\omega}$ there is retract $p: \kappa^{\omega} \longrightarrow M$ [12, Proposition 2.8]. In particular, $\ell K(Y) \leq \kappa$ if and only if there is an usco $\kappa^{\omega} \longrightarrow 2^{Y}$. The following two properties that we shall use can be found in [9] proven for the index $\ell \Sigma(X)$ but the proof for $\ell K(X)$ is completely analogous (as usual, $C_{p}(K)$ stands for the space of continuous functions over $K$ endowed with the pointwise convergent topology):

Proposition 7. Let $X$ be a Banach space and $K$ a compact space.

(1) If $Y$ is a closed subspace of $X$, then $\ell K(Y) \leq \ell K(X)$.

(2) If $Y$ is a total subset of $X$, then $\ell K(X) \leq \ell K(Y)$.

(3) If $Y$ is a subset of $C(K)$ which separates the points of $K$, then $\ell K(C(K))=$ $\ell K\left(C_{p}(K)\right) \leq \ell K\left(Y, \tau_{p}\right)$.

\section{The COMPACT Generation INDEX}

In this section we state some main properties of the index $C G(X)$ on Banach spaces which are analogous to well known properties of weakly compactly generated spaces. The first observation is that for a Banach space $X, C G(X)$ equals the least infinite number of weakly compact subsets of $X$ whose union is linearly dense in $X$, $X=\overline{\operatorname{span}} \bigcup_{\lambda<\kappa} K_{\lambda}$, since if $\bigcup_{i<\kappa} K_{i}$ is family of compacta whose union is linearly dense, we define $K_{F}=|F| \overline{c o} \bigcup_{i \in F} K_{i}$ for each finite subset $F$ of $\kappa$, and then we have another family of the same cardinality whose union is dense. In the following Theorem 8 we introduce and expose the main properties of $\kappa$-Eberlein compacta, which generalize well known facts about Eberlein ( $\omega$-Eberlein under this notation) compacta:

Theorem 8. Let $K$ be a compact space and $\kappa$ an infinite cardinal. The following are equivalent:

(1) $C G(C(K)) \leq \kappa$.

(2) $C G\left(C_{p}(K)\right) \leq \kappa$.

(3) $K$ is a subspace of a product $\prod_{\alpha<\kappa} K_{\alpha}$ of $\kappa$ many factors in which each $K_{\alpha}$ is an Eberlein compact.

(4) The space can be found as a subset $K \subset[0,1]^{\Gamma}$ so that $\Gamma=\bigcup_{\lambda<\kappa} \Gamma_{\lambda}$ and for every $x \in K$, and every $\lambda<\kappa$ the set $\left\{\gamma \in \Gamma_{\lambda}: x_{\gamma} \neq 0\right\}$ is finite.

When these conditions are satisfied, we say that $K$ is a $\kappa$-Eberlein compact.

Proof: That (1) implies (2) is clear since the weak topology is finer than the pointwise topology. That (2) implies (3): if $C G\left(C_{p}(K)\right) \leq \kappa$ then we have a family 
$\left\{S_{\lambda}: \lambda<\kappa\right\}$ of compact subsets of $C_{p}(K)$ whose union is dense in $C_{p}(K)$. In this situation each compact $K_{\lambda}$, defined as the quotient of $K$ by the relation $x \sim y$ iff $f(x)=f(y)$ for all $f \in S_{\lambda}$, is an Eberlein compact since $S_{\lambda}$ is a pointwise compact subset of continuous functions separates the points of $K_{\lambda}$ and on the other hand, $K$ is a subspace of $\prod_{\lambda<\kappa} K_{\lambda}$. That (3) implies (4) follows immediately from the well known fact, consequence of the Amir-Lindenstrauss Theorem [1, that $K$ is Eberlein compact if and only if it verifies (4) for $\kappa=\omega$. For (3) implies (1), $K$ is a subspace of $L=\prod_{\alpha<\kappa} K_{\alpha}$ where each $K_{\alpha}$ is Eberlein compact. Since $(C(K), w)$ is a continuous image of $(C(L), w)$ it is enough to see that $C G(C(L)) \leq \kappa$. For each finite subset $F$ of $\kappa$ we consider $K_{F}=\prod_{\alpha \in F} K_{\alpha}$ which is an Eberlein compact. The natural projection $L \longrightarrow K_{F}$ induces a one-to-one operator $T: C\left(K_{F}\right) \longrightarrow C(L)$, and since $C\left(K_{F}\right)$ is weakly compactly generated, $C G\left(T\left(C\left(K_{F}\right)\right)\right)=\omega$. The StoneWeierstrass theorem implies that $\bigcup\left\{T\left(C\left(K_{F}\right)\right): F \in[\kappa]^{<\omega}\right\}$ is dense in $C(L)$, so $C G(C(L)) \leq \kappa$.

Theorem 9. A Banach space $X$ is a subspace of a Banach space $Y$ with $C G(Y) \leq \kappa$ if and only if $\left(B_{X^{*}}, w^{*}\right)$ is $\kappa$-Eberlein.

PROOF: If $K=\left(B_{X^{*}}, w^{*}\right)$ is $\kappa$-Eberlein, then $C G(C(K)) \leq \kappa$ and $X$ is a subspace of $C(K)$. Conversely, let $\left\{Y_{\alpha}\right\}_{\alpha<\kappa}$ be a union of $\kappa$ weakly compactly generated subspaces of $Y$ which is linearly dense in $Y$. By the Theorem of Amir and Lindenstrauss [1], for every $\alpha$ there is a one-to-one weak ${ }^{*}$-to-pointwise continuous operator of norm $1, T_{\alpha}: Y_{\alpha}^{*} \longrightarrow c_{0}\left(\Gamma_{\alpha}\right)$ which induces by composition a weak*-topointwise continuous operator $T_{\alpha}^{\prime}: Y^{*} \longrightarrow c_{0}\left(\Gamma_{\alpha}\right)$. Finally, we have a one-to-one weak ${ }^{*}$-to-pointwise continuous function $\prod T_{\alpha}: Y^{*} \longrightarrow \prod c_{0}\left(\Gamma_{\alpha}\right)$ which implies that $B_{Y^{*}}$ is a $\kappa$-Eberlein compact in the weak* topology. Since $B_{X^{*}}$ is a continuous image of $B_{Y^{*}}$ it remains to show that:

Theorem 10. Any continuous image of a $\kappa$-Eberlein compact is $\kappa$-Eberlein compact.

Unlike the previous results, now Theorem 10 cannot be easily deduced from the well known (and difficult) particular case in which $\kappa=\omega$. We must instead adapt the arguments of the countable case to this more general context. We know at least two ways to do this but since we do not need here this result, and in any case its proof does not require really new ideas but just substituting some appearance of the countable power by an arbitrary $\kappa$, we just indicate how this can be done. One possibility is to follow the proof of [7] changing in the appropriate places the role of convergent sequences by nets indicated in the lattice of finite subsets of $\kappa$. For the other argument, we recall that a $\kappa$-Corson compact is a compact space which can be found as a subspace $K \subset \mathbb{R}^{\Gamma}$ such that for every $x \in K,\left|\left\{\gamma \in \Gamma: x_{\gamma} \neq 0\right\}\right| \leq \kappa$. It is a consequence of a result of Bell and Marciszewski [6], who generalized an argument by $\mathrm{Pol}$ [16, that the continuous image of a $\kappa$-Corson compact is $\kappa$-Corson. On the other hand, if in the terminology of [14] we define a $\kappa$-quasi-RN compact to be a compact whose diagonal is the intersection of $\kappa$-many almost neighborhoods of the diagonal, then following analogous arguments as in [4] and [14] it is possible to prove that the continuous image of a $\kappa$-quasi-RN compact is again $\kappa$-quasi-RN and that a compact space is $\kappa$-Eberlein if and only if it is $\kappa$-Corson and $\kappa$-quasi-RN. 
We point out that the mentioned result of [6] also says that $\ell\left(C_{p}(K)\right) \leq \kappa$ whenever $K$ is a $\kappa$-Corson compact and, as a consequence, if the dual unit ball of a Banach space $X$ is a $\kappa$-Corson compact in its weak* topology, then $\ell(X) \leq \kappa$.

\section{Adequate families}

The examples which we shall present will be based on adequate families of sets, a concept introduced by Talagrand 18, precisely to provide this kind of examples in the countable case. We state in this section, for the reader's convenience, the facts about this construction that we shall need. A family of subsets $\mathcal{A}$ of a given set $\Delta$ is called an adequate family if for every $A, A$ belongs to $\mathcal{A}$ if and only if every finite subset of $A$ belongs to $\mathcal{A}$. Associated to such a family, we have the compact space $K_{\mathcal{A}} \subset\{0,1\}^{\Delta}$ of the characteristic functions of elements of $\mathcal{A}$. The key fact proved by Talagrand is:

Theorem 11. If $\Delta$ is a topological space and $\mathcal{A}$ is an adequate family of subsets of $\Delta$ which consists only of closed subsets of $\Delta$, then there is an usco map $\phi: \Delta \longrightarrow 2^{X}$ with $X$ a point-separating subset of $C_{p}\left(K_{\mathcal{A}}\right)$.

Namely $X=\Delta \cup\{0\}$ is the point-separating subset of $C_{p}\left(K_{\mathcal{A}}\right)$ whose pointwise topology is the narrowest such that each set $A \in \mathcal{A}$ is closed and the usco map is $\Delta \longrightarrow 2^{X}$ given by $\delta \mapsto\{0, \delta\}$. Making use of Proposition 7 we get:

Corollary 12. If $\Sigma$ is a complete metric space of weight at most $\kappa$ and $\mathcal{A}$ is an adequate family of closed subsets of $\Sigma$, then $\ell K\left(C\left(K_{\mathcal{A}}\right)\right) \leq \kappa$.

We will also make use of the following fact:

Theorem 13. Let $\mathcal{A}$ an adequate family of subsets of a set $\Delta$ and suppose that $K_{\mathcal{A}}$ is a $\kappa$-Eberlein compact. Then, there exists a decomposition $\Delta=\bigcup_{\lambda<\kappa} \Delta_{\lambda}$ such that for every $x \in K_{\mathcal{A}}$ and every $\lambda<\kappa, x$ has only finitely many nonzero coordinates in $\Delta_{\lambda}$.

The proof is analogous to that of [11, Theorem 3.4.2], just changing countable families by families of cardinality at most $\kappa$ where necessary.

\section{WEAKLY LiNDELÖF DETERMINED SPACES OF ARBITRARILY HIGH COMPACT GENERATION INDEX}

In the following lemma, we state a standard fact of cardinal arithmetics which is important for all the discussions afterwards, namely that there are arbitrarily large cardinals $\tau$ with $\tau<\tau^{\omega}$.

Lemma 14. Let $\left\{\kappa_{n}\right\}_{n<\omega}$ be a sequence of cardinals such that $\kappa_{n+1}>\kappa_{n}$ for every $n<\omega$, and let $\tau$ be the supremum of this sequence. Then $\tau^{\omega}>\tau$. In particular, for any cardinal $\kappa$ there exists a cardinal $\tau>\kappa$ such that $\tau<\tau^{\omega}$.

Proof: Suppose that $\tau^{\omega}=\tau$ and let $f: \tau \longrightarrow \tau^{\omega}$ be a surjection from $\tau$ onto the set of sequences of elements of $\tau, \alpha \mapsto f(\alpha)=\left(f(\alpha)_{n}\right)_{n<\omega}$. For every $n<\omega$ the set $A_{n}=\left\{f(\alpha)_{n}: \alpha<\kappa_{n}\right\}$ has cardinality less than or equal to $\kappa_{n}<\tau$ hence we can choose $\beta_{n} \in \tau \backslash A_{n}$. The sequence $\beta=\left(\beta_{n}\right)_{n<\omega}$ is not in the image of $f$ which is a contradiction. The reason is that if $\beta=f(\alpha)$ for some $\alpha<\tau$, then there exists $n<\omega$ with $\alpha<\kappa_{n}$ and therefore $\beta_{n}=f(\alpha)_{n} \in A_{n}$ which is absurd. 
Proof of Theorem 2 2 It is a consequence of Lemma 14 that there exists a metric space $Z$ which cannot be expressed as the union of $\kappa$ many discrete subsets. Indeed, if $\kappa<\tau<\tau^{\omega}$, then $Z=\tau^{\omega}$ has this property because the weight of $Z$ is $\tau$ so its discrete subsets have cardinality less than or equal to $\tau$. From such a metric space $Z$, we shall construct a Corson compact space $K$ which is not $\kappa$-Eberlein. The construction is in a similar spirit as those carried out in [2] to give several examples of Corson compacta with specific properties. We consider a well order $<$ on $Z$. Set $\mathcal{A}$ the family of all subsets $A$ of $Z$ such that each finite subset is of the form $\left\{\xi_{1}<\ldots<\xi_{n}\right\}$ with $d\left(\xi_{i}, \xi_{j}\right) \leq \frac{1}{i}$ for $i<j$. Notice that this is an adequate family and that every set $A \in \mathcal{A}$ is either finite or countable. Indeed the order type of any $A \in \mathcal{A}$ in the well order $<$ cannot be greater than $\omega+1$ because if $\left\{\xi_{1}<\xi_{2}<\ldots<\xi_{\omega}<\xi_{\omega+1}\right\}$ belongs to $\mathcal{A}$, then $\xi_{\omega}=\lim _{n \longrightarrow \infty} \xi_{n}=\xi_{\omega+1}$ which is a contradiction. Hence, $K=K_{\mathcal{A}} \subset\{0,1\}^{Z}$ is a Corson compact and we shall see that it is not $\kappa$-Eberlein compact. Namely, if it were $\kappa$-Eberlein, by Theorem 13 there should be a decomposition $Z=\bigcup_{\lambda<\kappa} Z_{\lambda}$ such that each $A \in \mathcal{A}$ has only finitely many elements in each $Z_{\lambda}$. We can choose $\lambda$ with $Z_{\lambda}$ not discrete and take $z$ an accumulation point of $Z_{\lambda}$. We shall find an infinite subset of $Z_{\lambda}$ which belongs to $\mathcal{A}$ thus getting a contradiction. We take $\xi_{1}$ the first element of $Z_{\lambda}$ such that $\xi_{1} \in B(z, 1)$ (we are denoting by $B(x, \varepsilon)$ the open ball with center $x$ and radius $\varepsilon$ in the space $Z$ ). Second, we take $\xi_{2}$ the first element in $Z_{\lambda}$ greater than $\xi_{1}$ such that $\xi_{2} \in B\left(z, \frac{1}{2}\right) \cap B\left(\xi_{1}, 1\right)$. In the $n$-th step, if $\xi_{1}<\cdots<\xi_{n-1}$ have been defined we choose $\xi_{n}$ to be the first element of $Z_{\lambda}$ greater than $\xi_{n-1}$ such that

$\xi_{n} \in B\left(z, \frac{1}{n}\right) \cap \bigcap_{i=1}^{n-1} B\left(\xi_{i}, \frac{1}{i}\right)$. After this construction, $\left\{\xi_{n}: n<\omega\right\}$ is an infinite element of $\mathcal{A}$ inside $Z_{\lambda}$.

We set $X=C(K)$ with $K$ the compact space defined above. Since $K$ is not $\kappa$-Eberlein, $C G(X)>\kappa$. On the other hand, it has been proved in 3, Proposition 4.10] that if $K$ is a compact subset of $\{0,1\}^{\alpha}$ such that the order type of the supports of all elements of $K$ is uniformly bounded by a countable ordinal, then $C(K)$ is weakly Lindelöf determined.

Since always $C G(X) \leq 2^{\operatorname{Nag}(X)}$ and $C G(X) \leq \ell \Sigma(X)^{\omega}$, Theorem 2 also shows that there are weakly Lindelöf determined Banach spaces of arbitrarily large indices $\operatorname{Nag}(X)$ and $\ell \Sigma(X)$. The Banach spaces such that $\ell \Sigma(X)=\omega$ (or equivalently $\operatorname{Nag}(X)=\omega)$ are called weakly countably determined [18. Hence, for $\kappa \geq 2^{\omega}$, Theorem 2 provides examples of weakly Lindelöf determined Banach spaces which are not weakly countably determined and of Corson compact spaces which are not Gul'ko compact.

\section{The Relation Between the CompaCt generation indeX and the $\mathcal{K}$-ANALYTICITY INDEX}

Notice first that if a topological space $Y$ is union of $\tau$ many compacta $\left\{K_{\lambda}\right\}_{\lambda<\tau}$, then $\ell K(Y) \leq \tau$ because we can get an usco $\phi: \tau \longrightarrow 2^{Y}$ by $\phi(\lambda)=K_{\lambda}$. Using Proposition 7 we get as a consequence that for any Banach space $X$ :

$$
\ell K(X) \leq C G(X)
$$


On the other hand, if $\ell K(X) \leq \kappa$ then there is an usco $\phi: \kappa^{\omega} \longrightarrow 2^{X}$ and since the continuous image of a compact space by an usco is compact and $\kappa^{\omega}$ is the union of $\mathbf{d}(\kappa)$ many compacta,

$$
C G(X) \leq \mathbf{d}(\ell K(X))
$$

Finally, the last relation is that for any Banach space $X$,

$$
\operatorname{dens}(X) \geq \mathbf{b}_{\ell K(X)}(C G(X))
$$

We prove it by contradiction. Suppose the contrary and call $\delta=\operatorname{dens}(X)$, $\kappa=\ell K(X)$ and $\tau=C G(X)$ so that $\delta<\mathbf{b}_{\kappa}(\tau)$. We have an usco $\phi: \kappa^{\omega} \longrightarrow 2^{X}$ and we can find a subset $\Sigma \subset \kappa^{\omega}$ of cardinality $\delta$ such that $\phi(\Sigma)$ is dense in $X$. Since $\delta<\mathbf{b}_{\kappa}(\tau), \Sigma$ is a subset of a union of less than $\tau$ many compact subsets of $\kappa^{\omega}$, so $C G(X)<\tau$, a contradiction.

Relations (6.1) - 6.3) already prove one implication of Theorem 4 Before passing to the converse, we make an observation about the evaluation of the indices on a generalized Cantor cube: For $K=\{0,1\}^{\kappa}$ and $X=C(K)$ we have that $\ell(X)=\ell K(X)=C G(X)=\kappa$. On the one hand, clearly $\{0,1\}^{\kappa}$ is $\kappa$-Eberlein. On the other hand, the evaluation maps $D=\left\{\delta_{x}: x \in \kappa\right\}$ constitute a discrete pointwise closed subset of $C\left(\{0,1\}^{\kappa}\right)$, so $\ell(D)=\kappa$ and $\ell(X) \geq \kappa$.

Now we fix cardinals $\kappa, \tau$ and $\delta$ like in part (1) of Theorem 4 and we will show a Banach space like in part (2). First, we take $S$ a subset of $\kappa^{\omega}$ of cardinality $\mathbf{b}_{\kappa}(\tau)$ which can be decomposed into $\tau$ many pieces $S=\bigcup_{\lambda<\tau} S_{\lambda}$ verifying the following two properties:

(S.1) $S_{\lambda}$ is not contained in any union of less than $\tau$ many compacta of $\kappa^{\omega}$

(S.2) There is a subset $U \subset S$ of cardinality $\kappa$ such that $\left|U \cap S_{\lambda}\right| \leq 1$ for all $\lambda$ and such that $x_{0} \neq y_{0}$ for any two different elements $x, y \in U$.

We can construct such an $S$ as follows: Take $A$ a subset of $\kappa^{\omega}$ of cardinality $\mathbf{b}_{\kappa}(\tau)$ which cannot be covered by less than $\tau$ many compacta of $\kappa^{\omega}$ and $A^{\prime}=\left\{a_{\lambda}: \lambda<\tau\right\}$ a subset of $A$ of cardinality $\tau$. Without loss of generality, we suppose that the set $U=\left\{x \in \kappa^{\omega}: x_{0}=x_{n} \forall n<\omega\right\}$ of the constant sequences is a subset of $A^{\prime}$. For $\lambda<\tau$, we define $S_{\lambda}=\left\{x \in \kappa^{\omega}: x_{2 n}=\left(a_{\lambda}\right)_{n},\left(x_{2 n+1}\right)_{n \in \omega} \in A\right\}$ and $S=\bigcup_{\lambda<\tau} S_{\lambda}$.

We construct now compact space inspired on the example of Talagrand [18 of a Talagrand non Eberlein compact. Consider $\mathcal{A}$ the family of all subsets $A \subset S$ which verify the two following properties:

(A.1) There exists some $n(A)<\omega$ such that for every different elements $x, y \in A$, it happens that $x_{n(A)} \neq y_{n(A)}$ but $x_{m}=y_{m}$ for all $m<n(A)$.

(A.2) $\left|A \cap S_{\lambda}\right| \leq 1$ for every $\lambda<\tau$.

This $\mathcal{A}$ is an adequate family which consists of closed subsets of $S$, hence the compact space $L=K_{\mathcal{A}} \subset\{0,1\}^{S}$ is a $\kappa$-Talagrand compact, by Corollary 12. We define $Y=C(L)$, so that $\ell K(Y) \leq \kappa$. Indeed, $\ell K(Y)=\kappa$ because if $U$ is a set like in (S.2) then all subsets of $U$ belong to $\mathcal{A}$ and there is therefore a copy of $\{0,1\}^{\kappa}$ inside $L$ and, as we observed, $\ell K\left(C\left(\{0,1\}^{\kappa}\right)=\kappa\right.$. 
On the other hand, property (A.2) implies that $L \subset\{0,1\}^{S}$ is a $\tau$-Eberlein compact (indeed the partition $S=\bigcup_{\lambda<\tau} S_{\lambda}$ fulfills the conditions in Theorem $8(4))$ and hence, $C G(Y) \leq \tau$. We check now that precisely $C G(Y)=\tau$. Assume by contradiction that $L$ is $\tau^{\prime}$-Eberlein for some $\tau^{\prime}<\tau$. Then by Lemma 13 we would find a partition $S=\bigcup_{i<\tau^{\prime}} \Delta_{i}$ such that each element of $L$ has only finitely many nonzero coordinates in each $\Delta_{i}$. Let us analyze for a moment what this condition on $\Delta_{i}$ means. For a subset $F$ of $\kappa^{n}$, we denote $F \times \kappa^{>n}=\left\{x \in \kappa^{\omega}:\left(x_{0}, \ldots, x_{n-1}\right) \in F\right\}$ and if $G \subset \kappa^{m}$ with $m>n$ we write $F<G$ if the restrictions of the elements of $G$ to the first $n$ coordinates constitute precisely the set $F$. The fact that we cannot find an infinite subset $A$ of $\Delta_{i}$ satisfying (A.2) and also (A.1) with $\mathrm{n}(\mathrm{A})=0$ implies that there exist finite sets $F_{0} \subset \kappa$ and $G_{0} \subset \tau$ such that $\Delta_{i} \subset F_{0} \times \kappa^{>0} \cup \bigcup_{\lambda \in G_{0}} S_{\lambda}$. Analogously, paying attention in each step to sets $A$ with $n(A)=n$, we can find inductively for every $n<\omega$ finite sets $F_{n} \subset \kappa^{n}$ and $G_{n} \subset \tau$ such that $F_{n-1}<F_{n}$, $G_{n-1} \subset G_{n}$ and $\Delta_{i} \subset F_{n} \times \kappa^{>n} \cup \bigcup_{\lambda \in G_{n}} S_{\lambda}$. This implies that for every $i<\tau^{\prime}$ we can find a compact set $K_{i}=\bigcap_{n<\omega} F_{n} \times \kappa^{>n}$ of $\kappa^{\omega}$ and a countable set $G_{i} \subset \tau$ such that $\Delta_{i} \subset K_{i} \cup \bigcup_{\lambda \in G_{i}} S_{\lambda}$. Hence,

$$
S=\bigcup_{i<\tau^{\prime}} K_{i} \cup \bigcup_{\lambda \in \bigcup_{i<\tau^{\prime}} G_{i}} S_{\lambda}
$$

Since $\left|\bigcup_{i<\tau^{\prime}} G_{i}\right| \leq \tau^{\prime} \cdot \omega<\tau$, we can take $\lambda_{0} \notin \bigcup_{i<\tau^{\prime}} G_{i}$ and then $S_{\lambda_{0}}$ is covered by $\tau^{\prime}<\tau$ compact subsets of $\kappa^{\omega}$, a contradiction.

So far, we know that $\ell K(Y)=\kappa$ and $C G(Y)=\tau$. Since $|S|=\mathbf{b}_{\kappa}(\tau)$, this is the weight of $L \subset\{0,1\}^{S}$ (it could not be lower because of the general relation (6.3)), hence $\operatorname{dens}(Y)=\mathbf{b}_{\kappa}(\tau)$.

Finally, we consider the space $X=C(L) \oplus c_{0}(\delta)$. The space $c_{0}(\delta)$ is weakly compactly generated, so $C G\left(c_{0}(\delta)\right)=\omega=\ell K\left(c_{0}(\delta)\right)$ and $\operatorname{dens}\left(c_{0}(\delta)\right)=\delta$. All these indices, when considered on a finite product, take as value the maximum of the value of each factor, so $X$ is the space we were looking for.

\section{The number of COMPACT SPACES Which Generate A SUBSPACE}

This section is devoted to the proof of Theorem 3 The situation is very similar to the previous section. Let us assume that we are in the situation of part (2) of Theorem 3 . That $\tau \leq \delta$ is evident. Being $Y$ a closed subspace of $X$ then $\ell K(Y) \leq \ell K(X)$, and from this and (6.2), we have that

$$
\tau=C G(Y) \leq \mathbf{d}(\ell K(Y)) \leq \mathbf{d}(\ell K(X))=\mathbf{d}(\kappa),
$$

and since $\ell K(Y) \leq \kappa$, by (6.3),

$$
\delta=\operatorname{dens}(Y) \geq \mathbf{b}_{\ell K(Y)}(C G(Y))=\mathbf{b}_{\ell K(Y)}(\tau) \geq \mathbf{b}_{\kappa}(\tau) .
$$

For the converse, if $\tau<\min (\kappa, \delta)$, it is enough to take $X=C\left(\{0,1\}^{\kappa}\right) \oplus c_{0}(\delta)$ and $Y=C\left(\{0,1\}^{\tau}\right) \oplus c_{0}(\delta)$. So we assume from now on that $\kappa \leq \tau \leq \mathbf{d}(\kappa)$ and $\delta \geq \mathbf{b}_{\kappa}(\tau)$, and we will adapt an example of Argyros [11, Section 1.6] by similar modifications as in the proof of Theorem 4 . First, we take like in that proof a subset $S$ of $\kappa^{\omega}$ of cardinality $\mathbf{b}_{\kappa}(\tau)$ which can be decomposed into $\tau$ many pieces $S=\bigcup_{\lambda<\tau} S_{\lambda}$ verifying (S.1) and (S.2). We consider the compact space $K \subset[0,1]^{S}$ 
which consists of the function of the form $\frac{1}{n} \chi_{A}\left(\chi_{A}\right.$ is the characteristic function of the set $A$ ) for some natural number $n$ and some set $A$ satisfying:

(B.1) For every different elements $x, y \in A$, it happens that $x_{n} \neq y_{n}$ but $x_{m}=y_{m}$ for all $m<n$.

(B.2) $\left|A \cap S_{\lambda}\right| \leq 1$ for every $\lambda<\tau$.

We note that the decomposition $S=\bigcup_{t \in \kappa^{n}}\left\{\sigma \in S:\left.\sigma\right|_{n}=t\right\}$ verifies the conditions of Theorem 8 (4) for $\varepsilon=\frac{1}{n}$, so $K$ is a $\kappa$-Eberlein compact and since, again, it contains a copy of $\{0,1\}^{\kappa}, C G(C(K))=\kappa$. For every $\sigma \in S$ we consider the "projection" function $f_{\sigma} \in C(K)$ and we set $Y$ the subspace generated by $\left\{f_{\sigma}: \sigma \in S\right\}$. For every $\lambda<\tau$, it follows from condition (B.2), that $\left\{f_{\sigma}: \sigma \in S_{\lambda}\right\} \cup\{0\}$ is a pointwise (hence weakly) compact subset of $C(K)$. Therefore, $C G(Y) \leq \tau$. We suppose by contradiction that $C G(Y)=\theta<\tau$. The rest of the proof follows closely that of [11, Theorem 1.6.3]. As a consequence of the Amir-Lindenstrauss Theorem, we can find then a set of generators of $Y$ like $\left\{y_{\delta}: \delta \in \Delta\right\}$ such that $\Delta=\bigcup_{\eta<\theta} \Delta^{\eta}$ and $\left\{y_{\delta}: \delta \in \Delta^{\eta}\right\} \cup\{0\}$ is homeomorphic in the weak topology to the one compactification of a discrete set, being 0 the point of infinity. We define a function $F: S \times \Delta \longrightarrow \mathbb{R}$ like $F(\sigma, \delta)=y_{\delta}\left(\chi_{\{\sigma\}}\right)$.

Statement 1: For every $\sigma \in S, 1 \leq|\{\delta \in \Delta: F(\sigma, \delta) \neq 0\}| \leq \theta$. Namely, if that set were empty, since $\left\{y_{\delta}: \delta \in \Delta\right\}$ generates $Y$ that would mean that $y\left(\chi_{\{\sigma\}}\right)=0$ for all $y \in Y$ which is false for $y=f_{\sigma}$. On the other hand, since 0 is the "weak limit point" of $\left\{y_{\delta}: \delta \in \Delta^{\eta}\right\}$ each set $\left\{\delta \in \Delta^{\eta}:|F(\sigma, \delta)|>\frac{1}{m}\right\}$ is finite.

Statement 2: For every $\delta \in \Delta,|\{\sigma \in S: F(\sigma, \delta) \neq 0\}| \leq \omega$. Indeed, each $\left\{\sigma \in S:|F(\sigma, \delta)|>\frac{1}{m}\right\}$ is finite because we can find an element $y \in Y$ which is a linear combination of some $f_{\sigma^{1}}, \ldots, f_{\sigma^{k}}$ with $\left\|y-y_{\delta}\right\|<\frac{1}{2 m}$ and in this case, whenever $|F(\sigma, \delta)|>\frac{1}{m}$, it must be the case that $\sigma=\sigma^{i}$ for some $i \leq k$.

From these two statements, playing back and forth we find a partition $S=$ $\bigcup_{\alpha<\lambda} \Gamma_{\alpha}$ and disjoint sets $\Delta_{\alpha}$, all sets of cardinality at most $\theta$ such that whenever $F(\sigma, \delta) \neq 0$ then there exists $\alpha<\lambda$ such that $\sigma \in \Gamma_{\alpha}$ and $\delta \in \Delta_{\alpha}$.

Since $\left|\Gamma_{\alpha}\right| \leq \theta$ we can enumerate it as $\left\{\sigma_{\nu}^{\alpha}: \nu<\theta\right\}$ (with repetitions if necessary). Then, we set $\Sigma_{\nu}=\left\{\sigma_{\nu}^{\alpha}: \alpha<\lambda\right\}$ so that $S=\bigcup_{\nu<\theta} \Sigma_{\nu}$. Further, for $\nu<\theta, m \in \mathbb{N}$ and $\eta<\theta$ we set

$$
\Sigma_{\nu m \eta}=\left\{\sigma \in \Gamma_{\nu}: \exists \delta \in \Delta^{\eta}: y_{\delta}\left(\chi_{\{\sigma\}}\right)>\frac{1}{m}\right\} .
$$

By statement $1, \Sigma_{\nu}=\bigcup_{m \eta} \Gamma_{\nu m \eta}$ and moreover $S=\bigcup_{\nu<\theta, m<\omega, \eta<\theta} \Sigma_{\nu m \eta}$.

We proved in the previous section that for such a decomposition of $S$ into $\theta<\tau$ pieces, there must exist $\nu, m$ and $\eta$ such that $\Sigma_{\nu m \eta}$ is not contained in a compact subset of $\kappa^{\omega}$, and this implies that, for some $n<\omega$ there is an infinite set $A$ satisfying (B.1) and (B.2) which is contained in $\Sigma_{\nu m \eta}$, call it $A=\left\{\sigma_{i}: i<\omega\right\}$. Call $\alpha_{i}$ the only ordinal such that $\sigma_{i} \in \Gamma_{\alpha_{i}}$. Since $A \subset \Sigma_{\nu}, \sigma_{i}=\sigma_{\nu}^{\alpha_{i}}$ and if $i \neq j$ then $\alpha_{i} \neq \alpha_{j}$. Also, since $A \subset \Sigma_{\nu m \eta}$ for every $i$, there exists $\delta_{i} \in \Delta^{\eta}$ such that $y_{\delta_{i}}\left(\chi_{\left\{\sigma_{i}\right\}}\right)>\frac{1}{m}$. Notice that if $i \neq j$ then, $\delta_{i} \in \Delta_{\alpha_{i}}$ so $\delta_{i} \notin \Delta_{\alpha_{j}}$ and $y_{\delta_{i}}\left(\chi_{\left\{\sigma_{j}\right\}}\right)=0$. 
Let now $B$ be a finite subset of $A$. Then, for every $\sigma \in \Sigma$ we have

$$
f_{\sigma}\left(\frac{1}{l} \chi_{B}\right)=\frac{1}{l} \chi_{B}(\sigma)=\frac{1}{l} \sum_{\sigma^{\prime} \in B} \chi_{\left\{\sigma^{\prime}\right\}}(\sigma)=\frac{1}{l} \sum_{\sigma^{\prime} \in B} f_{\sigma}\left(\chi_{\left\{\sigma^{\prime}\right\}}\right) .
$$

Hence, $y\left(\frac{1}{l} \chi_{B}\right)=\frac{1}{l} \sum_{\sigma^{\prime} \in B} y\left(\chi_{\left\{\sigma^{\prime}\right\}}\right)$ for every $y \in Y$. Now let $B_{1} \subset B_{2} \subset \cdots$ be a sequence of finite subsets of $A$ whose union is $A$. Then $\frac{1}{l} \chi_{B_{j}} \longrightarrow \frac{1}{l} \chi_{A}$ and so

$$
\left|y_{\delta_{i}}\left(\frac{1}{l} \chi_{A}\right)\right|=\lim _{j \rightarrow \infty}\left|y_{\delta_{i}}\left(\frac{1}{l} \chi_{B_{j}}\right)\right|=\frac{1}{l}\left|y_{\delta_{i}}\left(\chi_{\left\{\sigma_{i}\right\}}\right)\right|>\frac{1}{l m}
$$

This is a contradiction since $\left\{\delta_{i}: i<\omega\right\} \subset \Delta^{\eta}$ so it weakly converges to 0 .

\section{REMARKS ON OTHER INDICES}

The only example that we know of a Banach space $X$ in which $\ell \Sigma(X)<\ell K(X)$ is one of Talagrand [19, a variation of which can be taken of density character $\omega_{1}$, cf. [5]. In this case $\ell \Sigma(X)=\omega<\omega_{1}=\ell K(X)$. The "enlargement" of such an example offers a number of difficulties and we do not even know whether there exists some Banach space $X$ with $\omega<\ell \Sigma(X)<\ell K(X)$.

About the Nagami index, it is provided in [9] an example of a topological space $Y$ with $\operatorname{Nag}(Y)<\ell \Sigma(Y)$. We shall provide next examples of this kind for spaces of the form $Y=C_{p}(K)$ with $K$ compact. This does not provide yet examples of Banach spaces for which the two indices do not coincide, because it is not clear whether the Nagami index coincides for the weak and the pointwise topology: the proof of the fact that $\ell \Sigma(C(K))=\ell \Sigma\left(C_{p}(K)\right)$ 9] heavily depends on the fact that closure points in metric spaces are limits of sequences.

Theorem 15. Let $\kappa$ be any infinite cardinal. Then there exists a compact space $K$ with $\operatorname{Nag}\left(C_{p}(K)\right) \leq \kappa<C G\left(C_{p}(K)\right)$.

We already observed that we always have $C G(X) \leq \ell \Sigma(X)^{\omega}$, so when $\kappa=\kappa^{\omega}$ the compactum of Theorem 15 verifies $\operatorname{Nag}\left(C_{p}(K)\right)<\ell \Sigma\left(C_{p}(K)\right)$.

Proof: Consider the space $T=\kappa^{\kappa}$ (the product of $\kappa$ many discrete spaces of size $\kappa)$ which has weight $\kappa$. We consider the adequate family $\mathcal{A}$ of all subsets $A$ of $T$ such that there is $\lambda<\kappa$ such that for any $x \neq y$ in $A,\left.x\right|_{[0, \lambda)}=\left.y\right|_{[0, \lambda)}$ and $x_{\lambda} \neq y_{\lambda}$. We take $K=K_{\mathcal{A}}$. We know from Theorem 11] that there is an usco $T \longrightarrow 2^{Y}$ with $Y$ a subset of $C_{p}(K)$ that separates the points of $K$. In an analogous way as it is proven for the index $\ell \Sigma(X)[9]$, this implies that $\operatorname{Nag}\left(C_{p}(K)\right) \leq \kappa$. Suppose now that $K$ were $\kappa$-Eberlein. Then, by Theorem 13 , we should be able to find a decomposition $T=\bigcup_{i<\kappa} T_{i}$ such that any set of $\mathcal{A}$ has only finitely many elements in each $T_{i}$. We will find $t \in T=\kappa^{\kappa}$ such that $t \notin T_{i}$ for any $i<\kappa$, thus obtaining a contradiction. We define it inductively. The set $\left\{x_{0}: x \in T_{0}\right\} \subset \kappa$ is finite so we may take $t_{0}$ out of it. This will guarantee that $t \notin T_{0}$. If we already defined $t_{j}$ for $j<i$, the set $\left\{x_{i}: x \in T_{i}\right.$ and $\left.x_{j}=t_{j} \forall j<i\right\}$ is finite, so we can choose $t_{i}$ out of it. This guarantees that $t \notin T_{i}$. 


\section{REFERENCES}

[1] D. Amir and J. Lindenstrauss, The structure of weakly compact sets in Banach spaces, Ann. of Math. (2) 88 (1968), 35-46.

[2] S. Argyros and S. Mercourakis, On weakly Lindelöf Banach spaces, Rocky Mountains J. Math. 23 (1993), no. 2, 395-446.

[3] S. Argyros, S. Mercourakis, and S. Negrepontis, Functional-analytic properties of Corsoncompact spaces, Studia Math. 89 (1988), 197-229.

[4] A. D. Arvanitakis, Some remarks on Radon-Nikodým compact spaces, Fund. Math. 172 (2002), no. 1, 41-60.

[5] A. Avilés, Radon-Nikodým compact spaces of low weight and Banach spaces, Studia Math. 166 (2005), no. 1, 71-82.

[6] M. Bell and W. Marciszewski, Function spaces on $\tau$-Corson compacta and tightness of polyadic spaces, Czechoslovak Math. J. 54(129) (2004), no. 4, 899-914.

[7] Y. Benyamini, M. E. Rudin, and M. Wage, Continuous images of weakly compact subsets of Banach spaces, Pacific J. Math. 70 (1977), no. 2, 309-324.

[8] M. R. Burke and M. Magidor, Shelah's pcf theory and its applications, Ann. Pure and Applied Logic 50 (1990), 207-254.

[9] B. Cascales, M. Muñoz, and J. Orihuela, Index of $\mathcal{K}$-determination of topological spaces, Preprint (2005).

[10] H. H. Corson, The weak topology of a Banach space, Trans. Amer. Math. Soc. 101 (1961), $1-15$.

[11] M. Fabian, Gâteaux differentiability of convex functions and topology, Canadian Mathematical Society Series of Monographs and Advanced Texts, John Wiley \& Sons Inc., New York, 1997, Weak Asplund spaces, A Wiley-Interscience Publication.

[12] A. S. Kechris, Classical descriptive set theory, Graduate Texts in Mathematics, vol. 156, Springer-Verlag, New York, 1995.

[13] M. Muñoz, Índice de $\mathcal{K}$-determinación de espacios topológicos y $\sigma$-fragmentabilidad de aplicaciones, Doctoral dissertation.

[14] I. Namioka, On generalizations of Radon-Nikodým compact spaces, Topology Proceedings (2001-2002), no. 26, 741-750.

[15] R. Pol, A function space $C(X)$ which is weakly Lindelöf but not weakly compactly generated, Studia Math. 64 (1979), no. 3, 279-285.

[16] - On weak and pointwise topology of function spaces, University of Warsaw preprint No. 4184, Warsaw (1984).

[17] H. P. Rosenthal, The heredity problem for weakly compactly generated Banach spaces, Compositio Math. 28 (1974), 83-111.

[18] M. Talagrand, Espaces de Banach faiblement $\mathcal{K}$-analytiques, Ann. of Math. (2) 110 (1979), no. $3,407-438$.

[19] _ A new countably determined Banach space, Israel J. Math. 47 (1984), no. 1, 75-80.

[20] E. K. van Douwen, The integers and topology, Handbook of set-theoretic topology, NorthHolland, Amsterdam, 1984, pp. 111-167.

Departamento de Matemáticas, Universidad de Murcia, 30100 Espinardo (Murcia), SPAIN

E-mail address: avileslo@um.es 